\title{
How Context-aware are Extant BPM Methods? - Development of an Assessment Scheme
}

\author{
Marie-Sophie Denner ${ }^{1}$, Maximilian Roeglinger ${ }^{1}$, Theresa Schmiedel $^{2}$, Katharina \\ Stelzl $^{1}$, Charlotte Wehking ${ }^{2}$ \\ ${ }^{1}$ University of Bayreuth, FIM Research Center, 95444 Bayreuth, Germany \\ ${ }^{2}$ University of Liechtenstein, 9490 Vaduz, Liechtenstein \\ \{sophie.denner, maximilian.roeglinger, katharina.stelzl\}@fim- \\ rc.de, \{theresa.schmiedel, charlotte.wehking\}@uni.li
}

\begin{abstract}
Context awareness is vital for business process management (BPM) success. Although many academics have called for context-aware BPM, current BPM research and practice do not seem to sufficiently account for various contexts. To examine whether this statement holds true, we developed an assessment scheme that enables determining to which extent existing BPM methods can be applied in various contexts. We identified 25 exemplary BPM methods based on a structured literature review and rated them according to their applicability to different context dimensions, i.e., goal, process, organization and environment dimension. Our results indicate that most BPM methods are rather context-independent, i.e., they are not geared to specific contexts. Accordingly, the investigated BPM methods follow a one-size-fits-all approach and practitioners have no guidance on how to tailor BPM in their organizations. In particular, there is a lack of BPM methods for explorative purposes as well as for knowledge- and creativity-intense business processes. In the digital age, which is characterized by volatility and high pressure for innovation, these domains are very important. Our research is a first step toward context-aware BPM methods and structured guidance for organizations regarding the systematic selection and configuration of BPM methods.
\end{abstract}

Keywords: BPM Methods, Context Awareness, Assessment Scheme, Literature Review

\section{Introduction}

In the last decades, business process management (BPM) has evolved into an important and mature domain in research and practice alike [49]. Organizations have increasingly adopted BPM in different contexts [19, 26, 52]. In fact, context awareness is one out of ten principles to efficiently and effectively use BPM in organizations [50]. Especially in the digital age, context awareness is one of the key characteristics of successful BPM [25]. As new technologies, customers' expectations, new business models, or additional competitors are hurdles that organizations currently need to overcome, organizations need to manage different contexts at the same time [20,25]. 
To adequately configure BPM with respect to the requirements of specific contexts, research has started to study context-aware BPM. Context awareness considers specific organizational factors that distinguish one organization from another based on given, situational, and organizational requirements [50]. According to the context framework by vom Brocke et al. [52], context-aware BPM considers the goal of BPM (i.e., exploration or exploitation), certain characteristics of the processes in focus (e.g., repetitiveness or creativity) as well as organizational (e.g., scope or culture) and environmental characteristics (i.e., uncertainty or competitiveness). While the process, organization, and environment dimensions indicate a given context and cannot be modified, the goal dimension can be actively chosen by the organization. An organization consciously decides whether its BPM should strive for exploitation (e.g., improvement), exploration (e.g., innovation), or both simultaneously.

To successfully institutionalize BPM, organizations can choose among a plethora of BPM methods [44, 48, 50]. Even though context awareness is critical for successful BPM, the current body of knowledge does not seem to account for business contexts $[45,52]$. One research stream generally addresses context awareness in BPM by investigating various context dimensions $[12,50]$. A second research stream focuses on context-aware methods by explicitly stating the application context of a BPM method $[1,13]$. Until now, only very few BPM methods seem to consider specific contexts. However, research mainly focuses on BPM methods by following a one-size-fits-all approach, not addressing context specifically [52]. For that reason, several researchers call for context-aware BPM as well as context-aware methods [29, 45, 48, 52]. Against this background, we investigate the following research question: How context-aware are extant BPM methods?

To answer this question, we provide an assessment scheme based on the context framework of vom Brocke et al. [52], which enables determining the context awareness of extant BPM methods. We set up the assessment scheme based on the four context dimensions of vom Brocke et al. [52]. Moreover, we identify 25 BPM methods based on a structured literature review. These BPM methods are exemplarily analyzed based on the assessment scheme by determining their applicability with respect to the four context dimensions. Finally, we reason about the context awareness of each dimension, context factor, and characteristic for all examined BPM methods. We also provide further insights into the sample at large. Being aware of the limitations of our literature review, we see our work as a first initial discussion of context-aware BPM methods. The assessment scheme is intended to serve as a starting point, offering guidance for BPM researchers to examine the context awareness of BPM methods. Additionally, the assessment scheme helps practitioners identify suitable BPM methods for specific contexts and goals.

Examining context awareness of existing BPM methods, we proceed as follows. Section 2 provides relevant theoretical background. In Section 3, we outline our data collection and analysis method. Section 4 presents and discusses the results of the applicability assessment concerning the identified BPM methods. We conclude in Section 5 by summing up the key results, discussing implications and limitations, and pointing to directions for further research. 


\section{Context-aware Business Process Management}

Organizations need to consider BPM in different contexts to perform efficiently and effectively [50]. The framework offered by vom Brocke et al. [52] helps organizations identify the context in which BPM is applied (Figure 1). Their framework consolidates a range of the latest research and serves as foundation for context-aware BPM research and practice. It includes four context dimensions, underlying context factors, and various related characteristics [52].

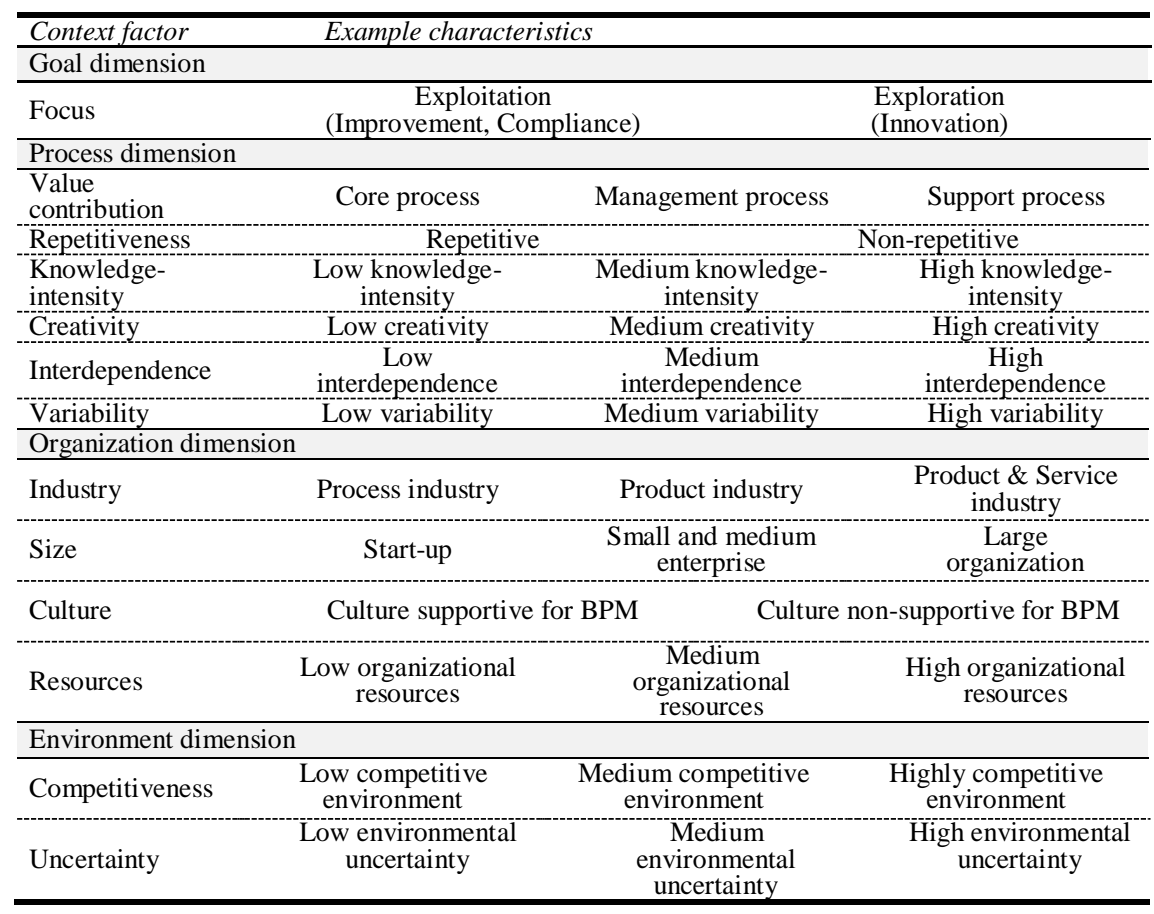

Fig. 1. Context Framework [52].

The goal dimension is crucial for BPM as it directly influences how BPM should be implemented and which methods should be applied [52]. Thereby, several authors recently differentiate between exploitation and exploration which is known as ambidextrous BPM [4, 29, 44]. Exploitative BPM is applied to realize incremental improvements by utilizing known methods [4, 44]. Explorative BPM gears to innovating processes by utilizing creative methods $[4,44]$. The process dimension includes various context factors to account for the diversity of processes and their requirements for an appropriate management [52]. Moreover, various context factors of an organization need to be considered, exemplary the size of an organization or the industry in which the organization operates [36, 42]. Finally, the environment dimension includes factors outside the organization, e.g., uncertainty in a rapidly changing environment or competition that influence the selection of BPM methods [43]. Accordingly, a context-aware method considers business process-relevant 
contextual information which influence the process goal [1, 13, 45]. Therefore, a context-aware BPM method explicitly states the application context [1, 13].

\section{$3 \quad$ Research Method}

\subsection{Identification of BPM Methods}

In this section, we first describe the process of collecting data via a structured literature review to systematically compile relevant BPM methods. Second, we explain our data analysis process (i.e., the structure of our assessment scheme) as well as the process of determining the context awareness of the identified BPM methods.

By conducting a structured literature review, we aim to identify extant BPM methods as a basis for further analysis. According to the nature of this research method, we explain all design decisions regarding suitable outlets, search strings, chosen time frame, and the selection process of relevant articles [51, 53]. We focused on the Business Process Management Journal (BPMJ) and the "Senior Scholars' Basket of Journals" as one of the most recognized outlets of the BPM and Information Systems (IS) discipline. Within the "Senior Scholars' Basket of Journals" [3] highly-rated journals with an eminent influence in the IS community, of which BPM is a part, are included. As for the BPMJ, we assumed that it discusses prevailing and core BPM research problems [9]. Nevertheless, we do not claim for completeness, as many other publication outlets could be included in our literature review. We critically reflect on the limitation in Section 5.1, pointing to further ideas for data collection. To cover articles dealing with extant BPM methods, we searched published articles full-text using the search strings summarized in Table 1. To avoid overlooking articles only referring to 'Business Process Management', we also included its abbreviation 'BPM'. Besides 'method', we included 'tool', 'model' and 'framework' as synonyms. As context-aware BPM has gained increasing attention in the past few years, especially with respect to various context dimensions [52] and the goal of ambidextrous BPM [29, 49], we confined the search to a time frame starting from 2014 to the present day.

Table 1. Overview of literature search approach.

\begin{tabular}{lr}
\hline Search string I & ("Business Process Management" OR "BPM") AND "method" \\
Search string II & ("Business Process Management" OR "BPM") AND "tool" \\
Search string III & ("Business Process Management" OR "BPM") AND "model” \\
Search string IV & ("Business Process Management" OR "BPM") AND "framework" \\
\hline
\end{tabular}

Having applied the search criteria to all selected journals, the list of search results contained 915 articles. Applying each search strings one after another, the number of search results included several duplicates, which we sorted out, and 255 unique articles remained. To ensure valid results, the final selection was conducted by three researches. We read the articles' titles and abstracts and removed 174 articles that did not match the scope of our research (e.g., article that only cited the BPMJ). The remaining 81 
articles were read in full and examined for their relevance to the research topic at hand. We eliminated articles with a descriptive purpose of use that do not develop a BPM method, but focused on case studies, statistical tests, development of capabilities, or a comparison of state of the art methods. Articles were considered relevant if they developed a BPM method. This led to a final removal of 56 articles and thus, the number of relevant articles ended up at 25. As the identified methods did not always have specific names, Table 2 lists the key idea and a short description of each method instead.

Table 2. List of all identified BPM methods.

\begin{tabular}{|c|c|}
\hline References & Key Idea (the BPM method helps organizations to...) \\
\hline [1] & identify contextual factors which impact processes and their process goals to adapt these \\
\hline [2] & assess the social sustainability of processes to diagnose participants resist following modelled process \\
\hline [5] & automatically detect potential process weaknesses in semantic process models \\
\hline [6] & $\begin{array}{l}\text { use modeling and simulation standards to measure process key performance indicators and test } \\
\text { improvements }\end{array}$ \\
\hline [7] & derive concrete recommendations for process improvement in a goal-oriented manner \\
\hline [8] & fit a probabilistic model according to a data set of past behavior base on predictive modeling \\
\hline [11] & assess the maturity of BPM governance practices to identify activities for improvement \\
\hline [14] & provide transparency concerning process ownership \\
\hline$[15]$ & $\begin{array}{l}\text { systematically and automatically analyze and match conceptual legacy process models in different } \\
\text { languages }\end{array}$ \\
\hline [17] & create value and improve efficiency based on analyzing strategic operations \\
\hline [18] & select the most suitable processes according to organizational objectives during a process \\
\hline$[21]$ & compile and structure organizational capabilities to facilitate and implement open innovations \\
\hline$[22]$ & facilitate organizational change through BPM \\
\hline$[24]$ & decompose BPMN models according to a structured guideline to improve process modelling \\
\hline [27] & reduce complexity of an initial BPMN model \\
\hline$[28]$ & achieve a process-oriented structure without destroying existing department structures \\
\hline$[30]$ & receive a value-oriented and holistic view of open innovation adoption inside the organization \\
\hline [32] & $\begin{array}{l}\text { build ambidexterity into inter-organizational IT-enabled service processes to meet the needs of their } \\
\text { customers }\end{array}$ \\
\hline [34] & $\begin{array}{l}\text { investigate their role in the value creation process by identifying potential value creation activities and } \\
\text { sources }\end{array}$ \\
\hline [38] & $\begin{array}{l}\text { provide an overview of process losses and corresponding prioritization steps for the elimination of such } \\
\text { losses }\end{array}$ \\
\hline [39] & extract business rules from existing process models \\
\hline [40] & $\begin{array}{l}\text { capture process knowledge to improve user collaboration and manage ad hoc and semi-structured } \\
\text { processes }\end{array}$ \\
\hline [41] & systematize operational processes for managing and improving processes \\
\hline [47] & $\begin{array}{l}\text { understand the customer needs and integrate the organizations' products and services into customer } \\
\text { processes }\end{array}$ \\
\hline [54] & extend context-aware process modeling towards location-awareness to increase organizational objectives \\
\hline
\end{tabular}

\subsection{Development of the Assessment Scheme}

To assess the applicability of extant BPM methods, we set up an assessment scheme based on the aforementioned context framework of vom Brocke et al. [52]. Originally, the context framework was developed for classifying the context of an organization in 
which BPM is applied. As BPM methods can help organizations to overcome the current hurdles of various contexts of an organization and facilitate the innovation or improvement of business processes [50], we classified the context awareness of BPM methods based on the context framework offered by vom Brocke et al. [52].

Structuring our analysis, we split the four context dimensions in the goal dimension, on the one hand, and the process, organization, and environment dimension on the other hand. As the goal dimension can be influenced by organizations, it commands for a conscious decision of the organization [52]. The goal dimension thus needs to be treated separately, as it is orthogonal to the other dimensions. Its characteristics (i.e., exploitation, exploration) build the columns of our assessment scheme. Contrary, the process, organization, and environment dimensions, which represent given context factors, build the lines of our assessment scheme. In case one of these context factors includes three characteristics, one of which reflects a medium level (e.g., medium knowledge-intensity), we decided to exclude the medium level. Besides being impractical for our purposes of assessing the context awareness of BPM methods, the medium level of a context factor is only qualitatively described and thus lacks a clear definition of the term 'medium'. Therefore, the medium level strongly depends on subjective interpretation that might bias the results.

Having set up the assessment scheme, the context awareness of each BPM method can be determined in three consecutive steps. First, the BPM method is analyzed regarding its goal, which means its applicability for exploitation and/or exploration. Thus, the BPM method is allocated to either or both columns of the assessment scheme. Second, regarding the other three context dimensions, the applicability of a BPM method for each specific characteristic is determined. Third, the context awareness of a BPM method is derived based on its applicability. Both, the applicability as well as the context awareness of a BPM method, are rated in terms of a Likert scale. The Likert scale is a scaling technique that can be used to obtain participants' level of agreement with given statements [33]. We used a three-point Likert scale, whereby its assessment criteria are interpreted as ordinal data with odd numbers [23]. Accordingly, the applicability of a BPM method regarding each characteristic in step two and step three, is specified by the following scale: (1) constitutes that the method is not applicable to a specific characteristic meaning the method is context-aware but does not support a specific characteristic, (3) constitutes that the method's applicability is independent of a specific characteristics meaning the method is context-independent, and (5) constitutes that the method is applicable to a specific characteristic meaning that the method is context-aware and supports a specific characteristic. Thus, all BPM methods assessed with either (1) or (5) are context-aware. A method assessed with (3) indicates an independence and neutrality concerning context awareness. This neutrality still implies an application of the method in an organization. Accordingly, a method assessed with either (1) or (5) is superior of (3) since the method is explicitly suitable for a specific context. However, applying a non-suitable method to a specific context is not recommendable. For example, if method A is applicable for a repetitive process, this characteristic is assessed with a value of (5) meaning that the method is contextaware and supports repetitive processes. If method A is not applicable for start-ups, this characteristic is assessed with a value of (1) meaning that the method is context-aware 
but does not support start-ups. If the applicability of method A is independent of a high knowledge-intensity, this characteristic is assessed with a value of (3) meaning that the method is context-independent and thus unaffected by knowledge-intense processes. To enable a more detailed discussion, we distinguish three levels of analysis based on the columns and lines of our assessment scheme. Based on the assessment of the applicability of each BPM method in step two, context awareness can be discussed with respect to each characteristic (first level of analysis). Additionally, context awareness is aggregated for all context factors (second level of analysis) as well as for the four context dimensions (third level of analysis). Thereby, the meaning of the assessment criteria (1), (3), and (5) is transferrable from the first to the second and third level of analysis. We further used statistics to calculate the relative frequency of each assessment criteria regarding each characteristic in percent $\left(f_{i}\right)$ and the median for each characteristic, context factor and context dimension $(m)$ (first level of analysis). The relative frequency indicates the number of times an assessment criterion occurred in relation to the number of all assessment criteria [16]. For instance, the relative frequency of (1) for the first level of analysis is calculated by the quantity (1) occurs for core processes divided through the quantity (1), (3), and (5) occurs for core processes.

To assess the extent of context awareness based on the relative frequency, we analyzed their tertile [16]. The tertile splits the distribution into three equal parts, whereas each part contains one third of the distribution. While a relative frequency below $33.3 \%$ represents weak context awareness, a relative frequency between $33.3 \%$ and $66.6 \%$ represents a moderate, and a relative frequency beyond $66.6 \%$ represents strong context awareness. The median splits the distribution of assessment criteria into halves and thus calculates the middle score of a ranked set of numbers. The median is calculated instead of the mean for ordinal data as it includes outliers in data [16]. For example, a median of three indicates that there are mostly context-independent BPM methods for one specific characteristic (e.g., support processes), a median of four indicates that BPM methods are moderately context-aware (e.g., management processes), and a median of five indicates that BPM methods are strongly contextaware (e.g., core processes).

To ensure the validity and reliability of assessments between two judges, different metrics are presented [37]. First, the validity of the assessments is verified by calculating hit ratios [37], whose values range from 1 for perfect agreement to 0 for perfect disagreement. Partial agreement is expressed via intermediate values. Considering the frequency of correctly assigned objects, validity is measured through method-specific and overall hit ratios [35]. To measure reliability, we used Cohen's Kappa [10], which mirrors "the proportion of joint judgement in which there is agreement after chance agreement is excluded" [37]. In cases of disagreement, the judges discuss all mismatching assessments and decide on one single statement (i.e., 1, 3 , or 5) in the end. 


\section{Context Awareness of BPM Methods}

\subsection{Application of the Assessment Scheme}

In this section, we first present the results of our assessment of 25 BPM methods and shortly discuss the inter-coder reliability. Second, we discuss the key findings with respect to context awareness of the investigated BPM methods.

Based on the results of our literature review and the developed assessment scheme, we determined the context awareness of 25 BPM methods. Therefore, two authors independently assessed the applicability of these methods regarding the goal dimension (step one) and the other three context dimensions (step two). Finally, the context awareness of the investigated BPM methods was derived (step three). The results are shown in Figure 2. If a BPM method is context-aware or context-independent regarding one specific characteristic, this is reflected by its assessment criteria (i.e., 1, 3, 5) as well as three corresponding colors.

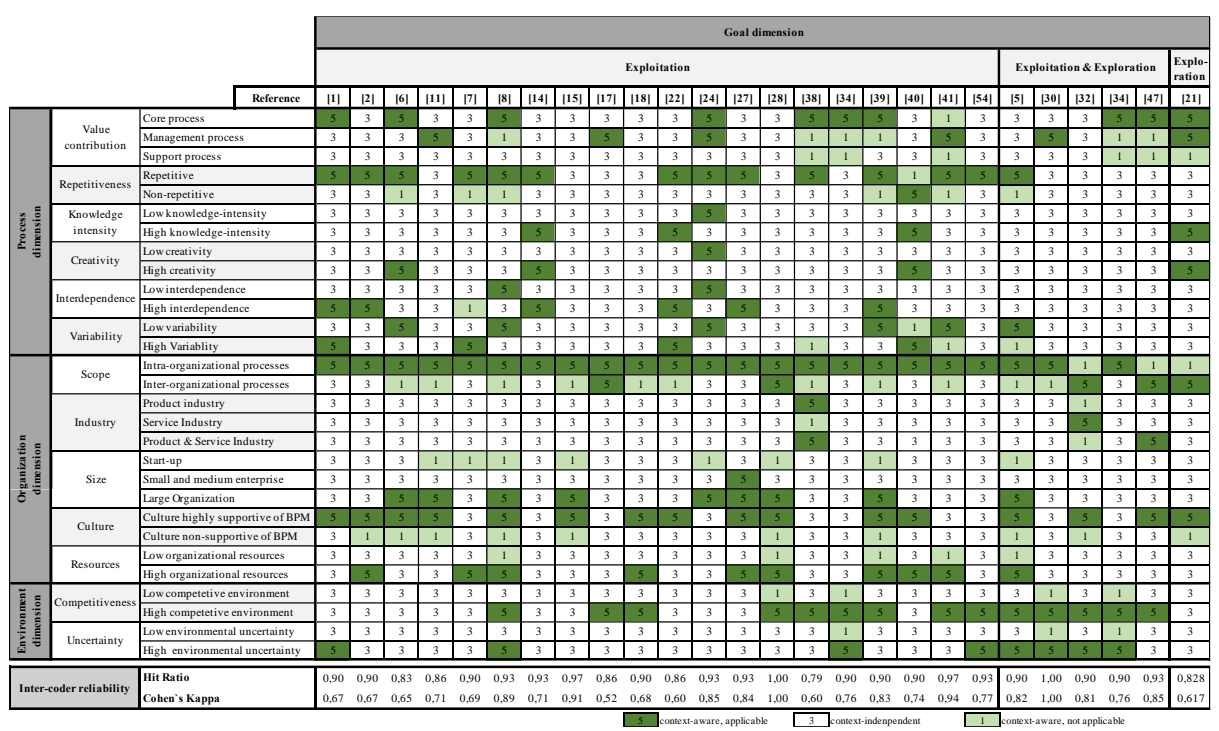

Fig. 2. Context awareness of extant BPM methods.

Moreover, the achieved method-specific hit ratios and the Cohen's Kappa coefficient for assessing inter-coder reliability are included in Figure 2. The two judging authors achieved method-specific hit ratios between 0.79 and 1.00 , yielding an overall average of $91 \%$ which was considered to represent a significant agreement [35]. Regarding Cohen's Kappa, the results ranged from 0.52 to 1.00 , reflecting the validity and reliability of our assessment [31]. 


\subsection{Discussion of Key Findings}

To enable a detailed discussion, we calculated the relative frequency in percent $\left(f_{i}\right)$ as well as the median $(m)$ for all levels of analysis based on our sample size. The results are shown in Figure 3. In the following, we discuss the results with respect to all levels of analysis. Therefore, we start with some overall findings regarding the relative frequency, before we analyze the four context dimensions (third level of analysis) in detail. Therefore, we present selected highlights for each context factor (second level of analysis) and all underlying characteristics (first level of analysis).

Overall findings. All in all, our results in Figure 2 and Figure 3 indicate that the investigated BPM methods are not yet aligned to various context dimensions. About $70 \%$ of the investigated BPM methods must be considered as context independent. This is supported by mainly medians of three. Only $30 \%$ of the investigated BPM methods are assessed with an assessment criterion of (1) or (5), which indicates context awareness. Moreover, it is interesting to note that $20 \%$ of the investigated BPM methods are context-aware and support specific characteristics (5), while only $10 \%$ especially address characteristics for which they are not applicable (1). However, characteristics for which a BPM method is not applicable are important to note as an application would probably lead to undesired results. For example, if a BPM method is developed only for service industries, an application in product industries is not

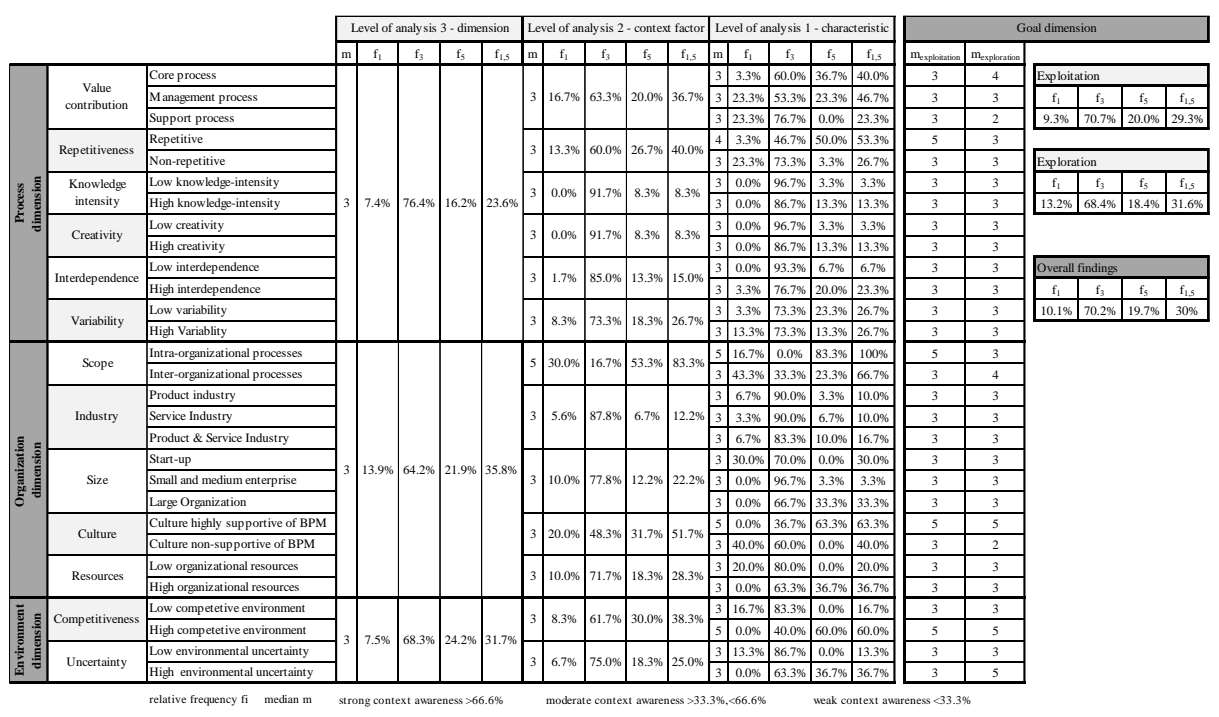

recommendable.

Fig. 3. Relative frequency and median for three levels of analysis.

Goal dimension. The assessment of the identified 25 BPM methods shows that 24 BPM methods are applicable for exploitation, six for exploitation and exploration, and one for exploration only. Thus, the ratio between BPM methods that focus on exploitation and exploration is unbalanced. 
Even though the BPM methods which refer to exploration are shorthanded, one third indicate context awareness $\left(f_{1,5}=31.6 \%\right)$. These six exploration BPM methods are especially applicable for core processes inter-organizational processes meeting the needs of customers in a highly competitive environment. To properly strive for explorative BPM, organizations also need a highly supportive BPM culture. Cultural values such as responsibility, excellence or customer orientation [46] build a solid foundation to successfully apply BPM methods for explorative purpose.

The 24 identified exploitation BPM methods indicate a slightly smaller degree of context awareness than those for exploration $\left(f_{1,5}=29.3 \%\right)$. Moreover, they are especially applicable for repetitive and intra-organizational processes in a highly competitive environment. As already mentioned for exploration, the support of BPM culture plays a crucial role to properly execute exploitation BPM methods.

Process dimensions. The third level of analysis shows that a quarter of all investigated BPM methods are context-aware referring to the process dimension $\left(f_{1,5}=23.6 \%\right)$. In particular, the second level of analysis illustrates that BPM methods are moderately context-aware regarding the context factors of value contribution and repetitiveness, while they are weak context-aware for knowledge-intensity, creativity, interdependence, and variability. The first level of analysis indicates that BPM methods for management and core processes are moderately context- aware, while for support processes the context awareness of BPM methods is weak. Moreover, no context-aware BPM methods are applicable for support processes $\left(f_{5}=0.0 \%\right)$. The same applied for the context factor of repetitiveness. More than half of all BPM methods indicate context awareness for repetitive processes which is also supported by a median of four. Further, the context awareness of BPM methods for non-repetitive processes is weak and most are not applicable for non-repetitive processes $\left(f_{1}=23.3 \%\right)$.

Organization dimension. Considering the third level of analysis, around one third of the investigated BPM methods are context-aware referring to the organization dimension $\left(f_{1,5}=35.8 \%\right)$. More precisely, for the context factors of scope and culture the context awareness of BPM methods is strong, while it is weak for industry, size, and resources (second level of analysis). BPM methods are strongly context-aware regarding the context factor of scope as $83.3 \%$ are applicable for intra-organizational processes (first level of analysis). This is also supported by a median of five. Only some BPM methods are context-aware and applicable for inter-organizational processes $\left(f_{5}=23.3 \%\right)$, especially if they focus on the goal dimension of exploration. This fact might be explained by an increased customer orientation along with BPM methods for exploration. Moreover, a median of five as well as two thirds of all identified BPM methods show that a highly supportive culture for BPM is required $\left(f_{5}=63.3 \%\right)$ (first level of analysis). Thus, a raising awareness regarding potentially neglected cultural factors is important to supplement existing BPM methods.

Environment dimension. For the environment dimension (third level of analysis), around one third of all BPM methods are context-aware $\left(f_{1,5}=31.7 \%\right)$, whereby the context awareness of BPM methods for the context factor competitiveness is greater than for uncertainty (second level of analysis). As a median of five and the relative frequency of high competitive environments $\left(f_{5}=60.0 \%\right)$ show, many BPM methods are especially applicable for high competitive environments. In most cases, also high 
environmental uncertainty requires context-aware BPM methods $\left(f_{5}=37 \%\right)$ (first level of analysis). 


\section{Conclusion}

\subsection{Summary and Implications}

In the digital age, organizations face several challenges such as fulfilling customers' changing wishes, facing high uncertainty or surviving in a dynamic competitive environment $[20,26]$. To overcome these challenges, organizations need to manage several contexts at the same time [50,52]. Given the increasing importance of context awareness and appropriate context-aware BPM methods for organizations in the digital age, the purpose of our study was to determine the context awareness of extant BPM methods regarding the four context dimensions (i.e., goal, process, organization and environment dimension) of the BPM context framework [52]. Therefore, we developed an assessment scheme for the context awareness of BPM methods which has been applied for the first time. Based on three levels of analysis, our results show many 'white boxes', which refer to a lack of context-aware BPM methods. In particular, BPM methods that focus on the goal of exploration seem to be rare. Further, our results suggest that BPM methods show room for improvement regarding context-awareness in the process, organization, and environment dimensions. Only very few methods account for these dimensions. Regarding the process dimension, particularly BPM methods geared toward the context factors knowledge-intensity, creativity, interdependence, and variability are seldom. Concerning the organizational dimension, size and industry have not been in focus yet. The context factor culture has been considered in most BPM methods, meaning that a supportive BPM culture is necessary to apply almost any BPM method. With reference to the environment dimension, BPM methods for the context factors of competitiveness and uncertainty need to be in the center of attention. Concluding, BPM research faces several gaps concerning context awareness and corresponding BPM methods. Research should strongly focus on appropriate methods which support different contexts. Emerging BPM methods should always consider and state the specific contexts they refer to.

The results of our research have implications for practice and research. The implications will be structured according to the assessment scheme and final results. Additionally, we point out how our work contributes to the research stream of contextaware BPM and ambidextrous BPM. First, with regard to theoretical implications, we developed an assessment scheme, which is based on the context framework of vom Brocke et al. [52]. The assessment scheme makes it possible to assess the context awareness of extant BPM methods based on three well-structured steps. Additionally, the assessment scheme offers a distinction in the goal dimension (horizontal axis) and the process, organization, and environment dimension (vertical axis). This distinction emphasizes that organizations can actively chose a BPM goal (i.e., exploitation or exploration), while the dimensions on the vertical axis are given and cannot be modified. Thus, an integrated perspective of context-aware and ambidextrous BPM is facilitated and allows for a detailed analysis of context dimensions that are given and influenceable. The assessment scheme also serves as a basis for further conceptual development (e.g., assigning BPM methods to a BPM lifecycle phase for further analysis, or developing a toolbox to assist organizations in systematically selecting 
context-aware BPM methods). Second, the final results of investigating 25 BPM methods point out a lack of context-aware BPM methods. Many 'white boxes' indicate that the majority of BPM methods are context-independent. Thus, our work serves as a starting point for further discussions and developments. We call for emerging contextaware BPM methods to manage several contexts simultaneously and meet the requirements of the digital age. Therefore, the assessment scheme facilitates the classification and development of a BPM method according to its context awareness in various dimensions.

With regard to the practical implications, the assessment scheme enables practitioners to assess extant BPM methods in a structured way. In particular, practitioners can check the applicability of currently used BPM methods and systematically select new ones. Especially the goal dimension underlines the importance of ambidextrous thinking, which means that an organization should focus on exploitation and exploration simultaneously and needs to consciously decide which goals require which BPM method. Thus, practitioners are able to improve the choice of BPM methods. Second, the final results illustrate that extant BPM methods are rarely context-aware. To be successful in the digital age, practitioners need to specifically select BPM methods from the limited number of context-aware BPM methods. Especially, if an organization strives for exploration, which is of utmost importance in the digital age, appropriate BPM methods need to be identified.

\subsection{Limitations and Further Research}

Being aware of the limitations of our research, we identified several directions for further research that are outlined in this section.

Our findings build on a few journals and a small sample size of BPM methods. We focused on specific journals such as the BPMJ or the "Senior Scholars' Basket of Journals" since they cover core BPM research. We do not claim for completeness and generalizability of all results. Yet, our results provide first insights about the context awareness of BPM methods. As direction for further research, we propose to broaden the sample size of BPM methods.

Another limitation is that we assessed the applicability of the BPM methods from a researchers' perspective only. We believe, however, that the current assessment is adequate to provide first insights on the context awareness of BPM methods. Further research may assess the applicability of BPM methods with both researchers and practitioners.

In the end, Table 3 presents our "Call for Action" for further research. On the one hand, the table summarizes ideas for further research based on the limitations mentioned above. On the other hand, it includes further research ideas beyond limitations. For instance, how the assessment scheme can be further developed or which potential issues could arise from dealing with context-aware BPM methods. Thus, Table 3 outlines different research areas, their corresponding research problems, and points to some theoretical and practical solution ideas. 
Table 3. "Call for Action" for further research.

\begin{tabular}{|c|c|c|}
\hline Area & Research Problem & Solution ideas \\
\hline \multirow{4}{*}{ 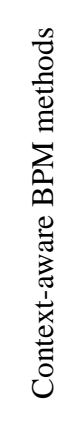 } & $\begin{array}{l}\text { Identify further BPM } \\
\text { methods }\end{array}$ & $\begin{array}{l}\text { - Search further literature (e.g., within BPM Handbooks or } \\
\text { conferences and journals covering BPM research) } \\
\text { - Include methods from other disciplines (e.g., innovation } \\
\text { management that may fit explorative purposes) }\end{array}$ \\
\hline & $\begin{array}{l}\text { Develop context-aware } \\
\text { BPM methods }\end{array}$ & $\begin{array}{l}\text { - Identify challenges for each context factor and develop } \\
\text { corresponding context-aware BPM methods (e.g., for } \\
\text { challenges of knowledge-intense processes) }\end{array}$ \\
\hline & $\begin{array}{l}\text { Support decision-making } \\
\text { for ambidextrous BPM }\end{array}$ & $\begin{array}{l}\text { - Develop a decision model that assists organizations in } \\
\text { selecting exploration and exploitation BPM methods }\end{array}$ \\
\hline & $\begin{array}{l}\text { Meet challenges of } \\
\text { realizing ambidextrous } \\
\text { BPM }\end{array}$ & $\begin{array}{l}\text { - Identify challenges that occur by simultaneously conducting } \\
\text { exploitation and exploration BPM methods } \\
\text { - Develop a maturity model that guides an organization to } \\
\text { realize ambidextrous BPM }\end{array}$ \\
\hline \multirow{4}{*}{ 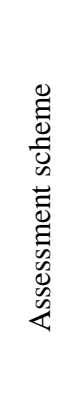 } & $\begin{array}{l}\text { Increase the number of } \\
\text { judges }\end{array}$ & $\begin{array}{l}\text { - Consult further BPM researchers and practitioners to } \\
\text { determine the applicability of extant BPM methods based on } \\
\text { our assessment scheme }\end{array}$ \\
\hline & $\begin{array}{l}\text { Extend the assessment } \\
\text { scheme }\end{array}$ & $\begin{array}{l}\text { - Add further context dimensions (i.e., a customer dimension } \\
\text { to better account for challenges in the digital age) } \\
\text { - Assign each BPM method to a BPM lifecycle phase for } \\
\text { further analysis (e.g.,process identification or analysis phase) }\end{array}$ \\
\hline & $\begin{array}{l}\text { Assess the current } \\
\text { context based on the four } \\
\text { context dimensions }\end{array}$ & $\begin{array}{l}\text { Operationalize each characteristic to assess the current } \\
\text { context of a process and an organization as a foundation to } \\
\text { select context-aware BPM methods }\end{array}$ \\
\hline & $\begin{array}{l}\text { Assist organizations in } \\
\text { selecting context-aware } \\
\text { BPM methods }\end{array}$ & $\begin{array}{l}\text { Develop a toolbox that enables organizations to assess their } \\
\text { context and decide which BPM methods are required for } \\
\text { which goal and lifecycle phases }\end{array}$ \\
\hline
\end{tabular}

\section{References}

[1] Anastassiu, M., Santoro, F. M., Recker, J., Rosemann, M.: The Quest for Organizational Flexibility: Driving Changes in Business Processes Through the Identification of Relevant Context. Business Process Mgmt Journal 22(4), 763790 (2016).

[2] Antunes, A. S., Rupino da Cunha, P., Barata, J.: MUVE IT: Reduce the Friction in Business Processes. Business Process Mgmt Journal 20(4), 571-597 (2014).

[3] Association for Information Systems: Senior Scholars' Basket of Journals. https:// aisnet.org/?SeniorScholarBasket, last accessed 2018/05/30.

[4] Benner, M. J., Tushman, M. L.: Exploitation, Exploration, and Process Management: The Productivity Dilemma Revisited. Academy of Mgmt Review 28(2), 238-256 (2003).

[5] Bergener, P., Delfmann, P., Weiss, B., Winkelmann, A.: Detecting Potential Weaknesses in Business Processes: An Exploration of Semantic Pattern Matching in Process Models. Business Process Mgmt Journal 21(1), 25-54 (2015).

[6] Bisogno, S., Calabrese, A., Gastaldi, M., Ghiron, N. L.: Combining Modelling and Simulation Approaches: How to Measure Performance of Business Processes. Business Process Mgmt Journal 22(1), 56-74 (2016). 
[7] Bolsinger, M., Elsäßer, A., Helm, C., Röglinger, M.: Process Improvement Through Economically Driven Routing of Instances. Business Process Mgmt Journal 21(2), 353-378 (2015).

[8] Breuker, D., Matzner, M., Delfmann, P., Becker, J.: Comprehensible Predictive Models for Business Processes. MISQ 40(4), 1009-1034 (2016).

[9] Business Process Management Journal: Business Process Management Journal. http://www.emeraldgrouppublishing.com/products/journals/ journals.htm?id=bpmj, last accessed 2018/05/30.

[10] Cohen, J.: A Coefficient of Agreement for Nominal Scales. Educational and Psychological Measurement 20(1), 37-46 (1960).

[11] de Boer, F. G., Müller, C. J., Schwengber ten Caten, C.: Assessment Model for Organizational Business Process Maturity with a Focus on BPM Governance Practices. Business Process Mgmt Journal 21(4), 908-927 (2015).

[12] de Bruin, T., Rosemann, M.: Towards a Business Process Management Maturity Model. In: Bartmann, D., Rajola, F., Kallinikos, J., Avision, D., Winter, R., Ein Dor, T., et al. (eds.) ECIS 2005 Proceedings of the $13^{\text {th }}$ European Conference on Information Systems, vol. n.d., pp. n.d., Regensburg, Germany (2005).

[13] Denner, M.-S., Püschel, L., Röglinger, M.: How to Exploit the Digitalization Potential of Business Processes. Business \& Information Systems Engineering, n.d.(n.d.), 1-19 (2017).

[14] do Prado Leite, J. C. S., Santoro, F. M., Cappelli, C., Batista, T. V., Santos, F. J. N.: Ownership Relevance in Aspect-Oriented Business Process Models. Business Process Mgmt Journal 22(3), 566-593 (2016).

[15] Fengel, J.: Semantic Technologies for Aligning Heterogeneous Business Process Models. Business Process Mgmt Journal 20(4), 549-570 (2014).

[16] Field, A.: Discovering Statistics using SPSS. $3^{\text {rd }}$ edn. Sage Publications Ltd., London (2009).

[17] Fiorentino, R.: Operations Strategy: A Firm Boundary-Based Perspective. Business Process Mgmt Journal 22(6), 1022-1043 (2016).

[18] Hakim, A., Gheitasi, M., Soltani, F.: Fuzzy Model on Selecting Processes in Business Process Reengineering. Business Process Mgmt Journal 22(6), 11181138 (2016).

[19] Harmon, P.,Wolf, C.: The State of Business Process Management 2014: A BPTrends Report. BPTrends (2014).

[20] Harmon, P.,Wolf, C.: The State of Business Process Management 2018: A BPTrends Report. BPTrends (2018).

[21] Hosseini, S., Kees, A., Manderscheid, J., Röglinger, M., Rosemann, M.: What Does It Take To Implement Open Innovation? Towards an Integrated Capability Framework. Business Process Mgmt Journal 23(1), 87-107 (2017).

[22] Inês Dallavalle de Pádua, S., Mascarenhas Hornos da Costa, J., Segatto, M., Aparecido de Souza Júnior, M., José Chiappetta Jabbour, C.: BPM for Change Management: Two Process Diagnosis Techniques. Business Process Mgmt Journal 20(2), 247-271 (2014).

[23] Jacoby, J., Matell, M. S.: Three-Point Likert Scales Are Good Enough. Journal of Marketing Research 8(4), 495-500 (1971). 
[24] Johannsen, F., Leist, S., Tausch, R.: Wand and Weber's Good Decomposition Conditions for BPMN: An Interpretation and Differences to Event-Driven Process Chains. Business Process Mgmt Journal 20(5), 693-729 (2014).

[25] Kerpedzhiev, G., König, U., Röglinger, M., Rosemann, M.: BPM in the Digital Age: BPM Capability Framework, http://digital-bpm.com/bpm-capabilityframework/, last accessed 2018/05/30.

[26] Kerpedzhiev, G., König, U., Röglinger, M., Rosemann, M.: Business Process Management in the Digital Age, http://digital-bpm.com/, last accessed 2018/05/30.

[27] Khlif, W., Ben-Abdallah, H., Ayed, N. E. B.: A Methodology For The Semantic and Structural Restructuring of BPMN Models. Business Process Mgmt Journal 23(1), 16-46 (2017).

[28] Khosravi, A.: Business Process Rearrangement and Renaming: A new Approach to Process Orientation and Improvement. Business Process Mgmt Journal 22(1), 116-139 (2016).

[29] Kohlborn, T., Mueller, O., Poeppelbuss, J., Roeglinger, M.: Interview with Michael Rosemann on Ambidextrous Business Process Management. Business Process Mgmt Journal 20(4), 634-638 (2014).

[30] Lamberti, E., Michelino, F., Cammarano, A., Caputo, M.: Open Innovation Scorecard: A Managerial Tool. Business Process Mgmt Journal 23(6), 1216-1244 (2017).

[31] Landis, J. R., Koch, G. G.: The Measurement of Observer Agreement for Categorical Data. Biometrics 33(1), 159-174 (1977).

[32] Lavikka, R., Smeds, R., Jaatinen, M.: A Process for Building Inter-Organizational Contextual Ambidexterity. Business Process Mgmt Journal 21(5), 1140-1161 (2015).

[33] Likert, R.: A Technique for the Measurement of Attitudes. Archives of Psychology 22(140), 1-55 (1932).

[34] Lindman, M., Pennanen, K., Rothenstein, J., Scozzi, B., Vincze, Z.: The Value Space: How Firms Facilitate Value Creation. Business Process Mgmt Journal 22(4), 736-762 (2016).

[35] Moore, G. C., Benbasat, I.: Development of an Instrument to Measure the Perceptions of Adopting an Information Technology Innovation. Information Systems Research 2(3), 192-222 (1991).

[36] Morton, N. A., Hu, Q.: Implications of the Fit Between Organizational Structure and ERP: A Structural Contingency Theory Perspective. International Journal of Information Mgmt 28(5), 391-402 (2008).

[37] Nahm, A. Y., Rao, S. S., Solis-Galvan, L. E., Ragu-Nathan, T. S.: The Q-Sort Method: Assessing Reliability And Construct Validity Of Questionnaire Items at a Pre-Testing Stage. Journal of Modern Applied Statistical Methods 1(1), 114125 (2002).

[38] Pereira Librelato, T., Pacheco Lacerda, D., Rodrigues, L. H., Veit, D. R.: A Process Improvement Approach Based on the Value Stream Mapping and the Theory of Constraints Thinking Process. Business Process Mgmt Journal 20(6), 922-949 (2014). 
[39] Polpinij, J., Ghose, A., Dam, H. K.: Mining Business Rules from Business Process Model Repositories. Business Process Mgmt Journal 21(4), 820-836 (2015).

[40] Rangiha, M. E., Comuzzi, M., Karakostas, B.: A Framework to Capture and Reuse Process Knowledge in Business Process Design and Execution Using Social Tagging. Business Process Mgmt Journal 22(4), 835-859 (2016).

[41] Rocha, R. d. S., Fantinato, M., Thom, L. H., Eler, M. M.: Dynamic Product Line for Business Process Management. Business Process Mgmt Journal 21(6), 12241256 (2015).

[42] Roeser, T., Kern, E.-M.: Surveys in Business Process Management - A Literature Review. Business Process Mgmt Journal 21(3), 692-718 (2015).

[43] Rogers, P. R., Miller, A., Judge, W. Q.: Using Information-Processing Theory to Understand Planning/Performance Relationships in the Context of Strategy. Strategic Mgmt Journal 20(6), 567-577 (1999).

[44] Rosemann, M.: Proposals for Future BPM Research Directions. In: Ouyang C., Jung JY. (eds.) Asia Pacific Business Process Management. AP-BPM 2014. Lecture Notes in Business Information Processing, vol. 181., pp. 1-15. Springer, Cham (2014).

[45] Rosemann, M., Recker, J., Flender, C.: Contextualisation of Business Processes. International Journal of Business Process Integration and Mgmt 3(1), 47-60 (2008).

[46] Schmiedel, T., vom Brocke, J., Recker, J.: Which Cultural Values Matter to Business Process Management? Business Process Mgmt Journal 19(2), 292-317 (2013).

[47] Trkman, P., Mertens, W., Viaene, S., Gemmel, P.: From Business Process Management to Customer Process Management. Business Process Mgmt Journal 21(2), 250-266 (2015).

[48] van der Aalst, W. M. P.: Business Process Management: A Comprehensive Survey. ISRN Software Engineering, 1-37 (2013).

[49] vom Brocke, J., Mendling, J.: Framework for Business Process Management: A Taxonomy for Business Process Management Cases. In: vom Brocke, J., Mendling, J. (eds.) Business Process Management Cases: Digital Innovation and Business Transformation in Practice, $1^{\text {st }}$ edn. Springer, Cham (2018).

[50] vom Brocke, J., Schmiedel, T., Recker, J., Trkman, P., Mertens, W., Viaene, S.: Ten Principles of Good Business Process Management. Business Process Mgmt Journal 20(4), 530-548 (2014).

[51] vom Brocke, J., Simons, A., Niehaves, B., Riemer, Kai, Plattfaut, R., Cleven, A.: Reconstructing the Giant: On the Importance of Rigour in Documenting the Literature Search Process. In: $17^{\text {th }}$ ECIS, pp. 2206-2217, Verona, Italy (2009).

[52] vom Brocke, J., Zelt, S., Schmiedel, T.: On the Role of Context in Business Process Management. International Journal of Information Mgmt 36(3), 486-495 (2016).

[53] Webster, J., Watson, R. T.: Analyzing the Past to Prepare for the Future: Writing a Literature Review. MIS Quarterly 26(2), xiii-xxiii (2002).

[54] Zhu, X., Recker, J., Zhu, G., Santoro, F, M.: Exploring Location-Dependency in Process Modeling. Business Process Mgmt Journal 20(6), 794-815 (2014). 